\title{
Mejoramiento genético en bovinos a través de la inseminación artificial y la inseminación artificial a tiempo fijo
}

\section{Genetic improvement in cattle through artificial insemination and artificial insemination at fixed time}

\author{
Melhoria genética em bovinos através de inseminação \\ artificial e inseminação artificial em horário fixo
}

\author{
Mayra Alejandra Marizancén Silva ${ }^{1}$ \& Lucerina Artunduaga Pimentel ${ }^{2}$ \\ ${ }^{1}$ Médico Veterinario Zootecnista, Especialista en Biotecnología Agraria; '2Zootecnista, Especialista en Alta Gerencia, \\ Magister en Educación con Énfasis en Educación Superior. \\ 1Programa Especialización en Biotecnología Agraria. ²Programa de Zootecnia, Escuela de Ciencias Agrícolas, \\ Pecuarias y del Medio Ambiente ECAPMA. Florencia, Caquetá, Colombia.
}

1/ucerina.artunduaga@unad.edu.co.2aleja1244@hotmail.com

\section{Resumen}

El sector ganadero busca mejorar la productividad de carne, leche y rusticidad, por medio de los cruzamientos, lo que ha conllevado al deterioro de las líneas raciales, repercutiendo en la disminución de calidad y cantidad de producción, e influyendo directamente en la rentabilidad. Actualmente los adelantos biotecnológicos proponen mejorar los niveles productivos de una empresa ganadera, a partir de la Inseminación Artificial IA y la Inseminación Artificial a Tiempo Fijo IATF, en donde se está manejando e introduciendo el mejoramiento genético, prácticas que incrementan el valor productivo y reproductivo de los bovinos, haciendo rentable el negocio ganadero y mejorando la competitividad del sector. La IA y la IATF, tienen como diferencia el tiempo del proceso de la inseminación, la IA se maneja a celo detectado y la IATF debe tener en cuenta las horas de la aplicación de las hormonas para la inseminación en tiempos exactos; estos dos métodos se manejan con el uso de semen probado y comprobado de animales altamente productivos para carne y/o leche.
Para la IATF se han proporcionado protocolos para la sincronización del estro basados en: "los que utilizan combinaciones de $\mathrm{GnRH}$ y prostaglandina $\mathrm{F} 2 \alpha$ (PGF), Ilamados protocolos Ovsynch y los que utilizan dispositivos con progesterona (P4) y estradiol conocidos como control del desarrollo folicular", división planteada por Saldarriaga (2009). En este escrito, se documenta la aplicabilidad de biotecnologías como la IA-IATF en los sistemas de producción ganaderos para contribuir al mejoramiento genético de las razas bovinas.

Palabras clave: inseminación artificial, inseminación artificial a tiempo fijo, mejoramiento genético.

\section{Abstract}

The livestock sector seeks to improve the productivity of meat, milk and rusticity, through crosses, which has led to the deterioration of racial lines, affecting the decrease in quality and quantity of production, and influencing directly the Profitability. Currently, 
biotechnological advances propose to improve the productive levels of a farming company, from artificial insemination Al and artificial insemination at fixed time IATF, where it is being managed and introduced genetic improvement, Practices that increase the productive and reproductive value of cattle, making the livestock business profitable and improving the competitiveness of the sector. Ai and IATF, have as a difference the time of the process of insemination, Al is managed to zeal detected and the IATF should take into account the hours of application of hormones for insemination in exact times; These two methods are handled with the use of tested and proven semen from highly productive animals for meat and/or milk. For IATF, protocols have been provided for Estrus synchronization based on: "Those using combinations of $\mathrm{GnRH}$ and prostaglandin F2 $\alpha$ (PGF), called Ovsynch protocols and those using devices with progesterone (P4) and estradiol known as Control of follicular development ", division raised by Saldarriaga (2009). In this paper, it is documented the applicability of biotechnology such as IA-IATF in livestock production systems to contribute to the genetic improvement of bovine breeds.

Key-words: artificial insemination, fixed-time artificial insemination, genetic improvement.

\section{Resumo}

O setor pecuário procura melhorar a produtividade da carne, do leite e da rusticidade, através de cruzamentos, o que levou à deterioração das linhas raciais, impactando na redução da qualidade $e$ quantidade de produção e influenciando diretamente a rentabilidade. Atualmente desenvolvimentos de biotecnologia visam melhorar os níveis de produção de uma empresa de gado, a partir da Inseminação Artificial Al e Inseminação Artificial Tempo Fixo IATF, onde eles estão dirigindo e introduzindo práticas de criação que aumentam o valor produtivo e reprodutivo o gado, tornando rentável o negócio de gado e melhorando a competitividade do setor. Al e IATF diferem no momento do processo de inseminação, $\mathrm{Al}$ é tratada com zelo detectado e a IATF deve levar em consideração as horas de aplicação dos hormônios para inseminação em momentos exatos; estes dois métodos são tratados com o uso de sémen provado e comprovado de animais altamente produtivos para carne e / ou leite. Para TAl eles forneceram protocolos para a sincronização do estro baseada na "aqueles que utilizam combinações de $\mathrm{GnRH}$ e da prostaglandina F2a (PGF), chamado protocolos Ovsynch e utilizando dispositivos com progesterona (P4) e desenvolvimento folicular controlo de estradiol conhecida ", Divisão proposta por Saldarriaga (2009). Neste documento, documentamos a aplicabilidade de biotecnologias, como IA-IATF em sistemas de produção pecuária, para contribuir para o melhoramento genético das raças bovinas.

Palavras chave: inseminação artificial, inseminação artificial de tempo fixo, melhoria genética.

\section{Introducción}

Biotecnologías como la Inseminación Artificial IA y la Inseminación Artificial a Tiempo Fijo IATF, han sido diseñadas para su aplicabilidad en el sector ganadero, principalmente para el mejoramiento genético. A través del tiempo la genética e identidad racial de los bovinos se ha perdido gracias a los cruzamientos entre animales, en búsqueda de mayores beneficios para el sector, en cuanto a productividad, rusticidad y adaptabilidad para las diferentes regiones, llegando a conformar animales multirraciales, designados como raza mestiza, en la cual se han deteriorado las características productivas principalmente de leche.

La IA, a través del uso del semen de toros altamente productivos resalta las características del padre, las cuales han sido evaluadas en varias generaciones ya sea en producción láctea y/o cárnica (Gasque, 2008). La introducción de este semen en el aparato reproductor de la hembra, con características raciales ya perdidas y con un nivel productivo promedio, concluye en una gestación que se desarrollará con 
las mejores características de sus padres, las cuales se verán reflejadas en el tiempo con nuevos cruzamientos y brindando mayor productividad.

La práctica de IA, es manejada directamente con la detección del celo y con el sistema AM-PM y PM-AM, las vacas que sean vistas en estro en la mañana, deben ser inseminadas durante la tarde del mismo día, y las vacas vistas en celo en la tarde, deben ser inseminadas después del amanecer del siguiente día (Foote, 2002), lo cual no determina con exactitud una gestación ya que se pueden presentar variaciones en el ciclo estral de cada hembra, el ambiente y el manejo. La técnica de la IA, según las experiencias se han ido mejorando en el tiempo, para evitar pérdidas económicas y disminuir los intervalos entre partos (IEP) en las ganaderías, a través del método IATF, el cual consiste en la sincronización del estro de la hembra con la aplicación de hormonas, respetando las horas y tiempo de los diferentes protocolos. Dentro de las propuestas biotecnológicas actuales y a través de este escrito, se pretende documentar la importancia de la aplicación de biotecnologías IAIATF, en los sistemas productivos ganaderos para contribuir al mejoramiento genético.

En América Latina, la actividad ganadera representa el uso más significativo de la tierra, siendo la actividad que más favorece y aporta al producto interno agropecuario de los diferentes países (Barragán, Mahecha \& Caja, 2015, citado por Molina, Sánchez, Uribe \& Atzori, 2016). El manejo de la ganadería a través del tiempo ha desarrollado en los bovinos la combinación de varias razas entre ellas, gracias al enfoque de los cruzamientos entre Bos taurus -razas productoras de leche- y Bos indicus -cebú, como productoras de carne-, en busca de un sistema de producción de doble propósito, de la rusticidad y adaptación al medio ambiente, llegando a perder la genética entre las líneas raciales y sus potenciales productivos en cuanto a carne, leche y crías al año, afectando al sector productor ganadero en niveles de calidad y cantidad, lo que influye directamente en la rentabilidad y perdidas económicas para los ganaderos por costos de producción de la crianza y mantenimiento de los animales en sus empresas ganaderas.
Investigaciones de Saldarriaga (2009), indican que en Colombia el uso de la inseminación artificial solo se implementa en un $20 \%$ de los sistemas productivos dedicados a la ganadería, el resto de los sistemas utiliza el sistema multitoro o también llamado monta natural. Actualmente la práctica de la IA-IATF está tomando acogida por parte de los grandes productores, principalmente por el nivel de adquisición a las propuestas biotecnológicas y a las exigencias comerciales y productivas, lo cual interviene para garantizar la productividad ganadera de carne y leche de calidad a partir del mejoramiento genético, influyendo directamente en la competitividad del sector.

La falta de información y divulgación de estas prácticas ante los pequeños productores ha limitado un poco el nivel productivo del sector ganadero, principalmente por el desconocimiento de la relación costo-beneficio, lo cual incide directamente en la continuidad de la producción y reproducción de forma tradicional, conllevando a la comercialización de productos de baja calidad, mayor tiempo de producción y poca rentabilidad. Colombia busca reconocimiento como líder en el desarrollo, producción, comercialización y exportación de productos de alto valor agregado, siendo el objetivo para 2025, posicionarse como uno de los tres líderes del sector biotecnológico en América Latina (Montenegro \& Hernández, 2015).

El desarrollo de la biotecnología reproductiva involucra el mejoramiento genético para rescatar las potencialidades productivas a través de la IA y la IATF lo cual incide en mejorar la genética a partir de la reproducción y su producción por medio del manejo del ciclo estral de la vaca, garantizando una cría al año y así rescatar las características productivas en el tiempo, de generación en generación con el uso de reproductores superiores a través del semen a partir de los cruzamientos interraciales y selección genética (Carvajal \& Kerr, 2015).

\section{Mejoramiento genético}

En la actualidad los programas de mejoramiento genético han desarrollado estrategias biotecnológicas como la IA y la IATF para la obtención de animales 
doble propósito -producción de carne y leche-, a partir de los cruzamientos interraciales y selección genética. Carvajal \& Kerr (2015), afirman a través de estudios que la composición de la leche es regulada por varios factores siendo uno de los más importantes la genética, y para el ganado de carne en cuanto a la ganancia de peso a través de la IA, se pueden hacer los cruzamientos precisos para proveer tasa de crecimiento y musculatura a las crías con el uso del semen seleccionado, generando un animal para el mercado, producido en forma rentable.

En estudios genéticos, se ha establecido como manejo principal la identificación de los mejores animales, los cuales deben ser seleccionados y usados como los padres de la próxima generación, compartiendo la opinión de Uribe (s.f,) quien afirma "La inseminación artificial juega un rol preponderante en la diseminación de genes deseables en una población". Cabe resaltar, que la genética y el principio del mejoramiento se basa principalmente en la producción: "PRODUCCIÓN = GENOTIPO + MEDIO AMBIENTE" (Ruiz, 2013), el cual debe tenerse en cuenta para la su aplicación e implementación en las empresas ganaderas según su sistema de producción -carne, leche, doble propósito-, para así mejorar y competir en el sector productivo. También es importante resaltar que las prácticas de mejoramiento genético están directamente relacionadas con el manejo -Buenas Prácticas Ganaderas-BPG-, el estado de las hembras y con las labores de administración a través del manejo de registros, lo cual puede repercutir favorable o desfavorablemente a la hora de la concepción luego de la aplicación de la IA-IATF y de la rentabilidad de los resultados:

\section{Buenas Practicas Ganaderas BPG}

Las BPG constituyen una guía de recomendaciones técnicas aplicadas en los sistemas ganaderos con el fin de garantizar la inocuidad de la producción -carne y/o leche-. Según las definiciones de Gambini (2009), categoriza las BPG en tres principios u objetivos: "higiene e inocuidad alimentaria, cuidado del medio ambiente y velar por la seguridad y salud de las personas -consumidores y trabajadores-", este concepto es direccionado principalmente a la comercialización y rentabilidad del sector ganadero. Cabe resaltar que el manejo y aplicabilidad de estas técnicas aportan directamente beneficios al bienestar y salud animal, mejorando las condiciones fisiológicas, metabólicas y de estrés, que repercuten en la producción y reproducción.

\section{Factores que afectan la fertilidad en las hembras}

- Condición corporal (CC): según definiciones de Bavera \& Peñafort (2005) la condición corporal se asimila al de estado corporal, es decir, al nivel de reservas corporales que el animal dispone para cubrir los requerimientos de mantenimiento y producción, indicando el estado nutricional y de salud del animal, la cual es medida por escalas de 1 a 5 o 1 a 9 según las definiciones del productor y/o veterinario (Tabla 1), estas dos escalas manejan el mismo criterio de evaluación en el animal , en donde 1 se caracteriza por ser demasiado flaca o caquéxica y 5 o 9 por ser un animal obeso o gordo. En ambos rangos la fertilidad es nula o limitada ya que su sistema reproductivo y endocrino -hormonal- no está apto para manejar el ciclo estral, atender una gestación y/o mantener un nivel de producción adecuado.

Tabla 1. Correlación entre las escalas 1 a 5 y 1 a 9 para CC.

\begin{tabular}{c|c|c|c|c|c|c|c|c|c}
\hline Escala & \multicolumn{10}{c}{ Grados } \\
\hline $\mathbf{1}$ a $\mathbf{5}$ & 1 & 1.5 & 2 & 2.5 & 3 & 3.5 & 4 & 4.5 & 5 \\
\hline $\mathbf{1}$ a 9 & 1 & 2 & 3 & 4 & 5 & 6 & 7 & 8 & 9 \\
\hline
\end{tabular}

Fuente: Bavera \& Peñafort (2005) 
- Estrés calórico: Son los problemas metabólicos ocasionados por el aumento de la temperatura corporal del animal generado por la combinación de temperatura ambiente y humedad relativa (Araujo, 2011). Esta condición afecta el consumo, actividad física, producción de leche, ciclo estral y reproducción en las vacas. Caraviello (2004), manifiesta que "un incremento en la temperatura corporal al momento de la inseminación deriva en baja fertilización y alta incidencia de muerte embrionaria, porque la viabilidad del oocito, esperma y embrión se ven comprometidas".

- Nutrición: afecta directamente aspectos fisiológicos y reproductivos en las hembras principalmente en estado productivo; "la cantidad de alimento ingerido y la fuente de energía afectan las variables del ciclo estral, como duración, patrones de ondas foliculares, tamaño de las estructuras ováricas y concentraciones circulantes de hormonas esteroideas" (Santori, 2009). Nutrimentos energéticos y minerales como selenio, cobalto, fósforo, hierro, cobre y yodo, se encuentran relacionados directamente con el sistema reproductivo y sus deficiencias afectan las concentraciones hormonales que intervienen en el ciclo estral.

\section{Manejo de registros}

La producción ganadera en estos tiempos se determina como una empresa, en la cual el productor debe manejar datos e información recogida en forma permanente de la producción de su ganadería, permitiendo de esta forma hacer un análisis financiero para establecer la rentabilidad de la inversión. Según Donato (s.f.), la empresa ganadera establece como unidad de producción la tierra, en donde es importante determinar cuál fue la producción de carne y/o leche por hectárea/año para evaluar la rentabilidad de la misma.

- La información más importante para tener en cuenta en una producción ganadera:

- Presentación de celos.

- Aplicación de la IA.

- Nacimientos.

- Producción de leche.
- Ganancia de peso.

- Indicadores reproductivos y control reproductivo -palpaciones-

El manejo de registros y su evaluación no solo demuestran el estado de pérdidas o ganancias de la empresa ganadera, sino que aporta criterios para hacer cambios y así proyectar el futuro de la explotación.

\section{Indicadores reproductivos}

A partir de los registros reproductivos se deben evaluar las diferentes situaciones encontradas en cada una de las hembras, lo cual indicara la selección, aplicación de tratamientos o descartes de las mismas, como:

- Días abiertos DA: Los días abiertos de las vacas comprenden el tiempo entre el parto y el momento en el cual la hembra vuelve a quedar preñada (Donato, s.f).

- Intervalo entre parto IEP: Es el tiempo que transcurre entre dos partos (Donato, s.f.).

- Intervalo parto primer celo/servicio: Es el número de días que pasan entre el último parto y la presentación del celo, tiempo ideal para la inseminación artificial o el primer servicio cuando se trata de monta natural (Donato, s.f).

- Tasa de natalidad: Donato (s.f), conceptualiza el parámetro hacia la práctica de la IA, y lo establece, al dividir el número de nacimientos entre el total de hembras aptas y sincronizadas para la reproducción que conforman la empresa ganadera.

- Índice de no retorno: indican el porcentaje de vacas que no repiten celo o que no repiten servicio en un periodo determinado (Gallego, 1998).

\section{Patrimonio genético en Colombia}

Los países en vía de desarrollo como Colombia, presentan en la ganadería un estancamiento a nivel biotecnológico, debido a la poca inversión del sector rural, altos costos de insumos y medicamentos veterinarios, baja cobertura educativa, capacitaciones y de información, vías de acceso en mal estado -vías terciarias-, falta de electrificación y TIC'S, mínimos incentivos productivos -valor de la leche y carne-, poca inversión en investigación a comparación de países como Estados Unidos, Brasil, Argentina, 
Chile, México, en donde el apoyo al sector agropecuario por parte del gobierno es fundamental para las políticas de desarrollo productivo.

Información en el Plan Estratégico de la Ganadería Colombiana (PEGA) 2019 por parte de FEDEGAN-FNG (2006), reporta 23 millones de bovinos de diferentes razas y cruces, de los cuales el $72 \%$ corresponde a ganado Bos indicus (cebuinos), el $15 \%$ a Bos taurus (razas europeas) $y$ el $13 \%$ a las conocidas como razas criollas y sus cruces. A nivel comercial cárnico se cuenta actualmente con indicadores en combinación entre edad y peso al sacrificio, situándose en un promedio de cuatro años con un peso aproximado de $400 \mathrm{~kg}$, con una ganancia de peso de $350 \mathrm{~g} / \mathrm{d}$ ía, a comparación de Argentina que maneja tiempo de sacrificio a los tres años con ganancia de peso de $550 \mathrm{~g} /$ día, factores que inciden directamente en la rentabilidad de producción. Por otra parte la información de producción láctea de nuestro país oscila alrededor de $4.5 \mathrm{~L} / \mathrm{vaca} /$ día en comparación con algunos referentes internacionales como Argentina y Uruguay, que reportan alrededor de 13 L/vaca/día y Estados Unidos con $25 \mathrm{~L}$ (FEDEGAN-FNG, 2006).

Fuentes (s.f), presenta las cifras de inseminación bovina a nivel internacional: Estados Unidos 90\%, Argentina $80 \%$, Brasil $80 \%$ y Perú $15 \%$, con el fin elevar los niveles de mejoramiento genético y productividad y en estudios y análisis de Galeano (2010), presenta a países como México, Chile y Argentina, con avances en los programas de mejora genética animal centrados en la estimación de parámetros genéticos y fenotípicos para las diferentes características de interés económico en los sistemas de producción de carne y leche, indicadores para la selección y determinación de la IA-IATF, en pro del desarrollo productivo.

\section{Sanidad animal a nivel reproductivo}

El manejo de estas biotecnologías está interviniendo directamente en la sanidad animal a nivel reproductivo disminuyendo o anulando definitivamente enfermedades como brucellosis, leptospirosis, herpesvirus bovino-1, diarrea viral bovina,
Neospora caninum (Motta et al, 2012), y otras como tricomoniasis y campylobacteriosis (Campero, 2000), a diferencia del manejo de monta natural donde se pueden transmitir este tipo de enfermedades, las cuales van a influir directamente en la rentabilidad de las producciones, ya que causan abortos, infertilidad, disminución de la producción, los animales se convierten en transmisores potenciales, principalmente por el toro o las secreciones expulsadas con carga bacteriana, vírica o parasitaria. Estas enfermedades no solo afectan la salud animal y sus producciones si no también la del hombre ya que algunas se caracteriza por ser zoonóticas, como en el caso de la brucelosis y leptospirosis.

\section{Método de inseminación artificial (IA)}

La práctica de la inseminación artificial, se maneja a través de la introducción de semen de toros genéticamente seleccionados con alto potencial productivo, a los cuales se les ha recolectado el semen por distintos métodos, el cual permanece conservado hasta el momento de su utilización.

Según la descripción de Fuentes (s.f.), la detección del celo para el manejo de la inseminación artificial es el indicador de éxito en campo, la cual depende de la detección adecuada del estro y de la habilidad en la inseminación. El principio clásico para la IA es el sistema AM - PM y PM - AM, las vacas que sean vistas en estro en la mañana, deben ser inseminadas durante la tarde del mismo día, y las vacas vistas en celo en la tarde, deben ser inseminadas después del amanecer del siguiente día (Foote, 2002). Todo esto basado en la observación, la palpación de ovarios y los datos sobre servicios/monta.

Descripciones de Restrepo (2008), indica las principales ventajas que posee la IA para su aceptación entre los productores: el bajo costo del semen y su aplicación y el éxito que garantiza el proceso, también el menor costo del servicio, menores riesgos asociados con el uso del toro, una mayor ganancia genética, y tasas de preñez que pueden ser mejores respecto a la monta natural. 


\section{Método de inseminación artificial a tiempo fijo (IATF)}

La IATF, involucra que las hembras se deben manejar con hormonas para sincronizarlas y modificar el comportamiento del sistema reproductivo, preparándolas para la recepción del semen y así lograr la gestación.

Martínez (s.f.), describe la IATF como: Un protocolo manejado para manipular hormonalmente a la vaca para que ovule el día que se programe y conceptualiza para su manejo que: en este protocolo es muy importante entender cómo funciona la fisiología reproductiva de la vaca, puesto que aunque existe una gran variedad de productos y de protocolos, se debe escoger el más adecuado según el caso -novilla, vaca, postparto, carne, leche-. También se indica que para dicho manejo se recomienda seleccionar un grupo de animales, sincronizarlos e inseminarlos el mismo día, a partir de la manifestación o detección de celos.

\section{Hormonas para los protocolos de la IATF}

Martínez (s.f.) referencia, las hormonas a tener en cuenta al momento de aplicar la IATF:

- GnRH: Induce la liberación de hormonas como FSH y LH, las cuales inciden en la regulación del desarrollo folicular. Se recomienda usarla en protocolos para vacas paridas postparto con buena condición corporal.

- Progestágenos: Vienen en implantes de liberación lenta, ya sean vaginales o en la oreja. El objetivo de usar los implantes es simular un diestro para controlar la ovulación hasta que se retire el mismo.

- Prostaglandinas PGF: Se usan en cualquier protocolo, su función principal es destruir el cuerpo lúteo terminando con la fase luteal (luteolítico), son los más usados en programas de sincronización de celos, ya que un 50 a 70\% de los animales tratados responderán con la presentación de celo y ovulación dentro de los 6 días siguientes a una aplicación de PGF, cuya fertilidad es igual o superior a una ovulación espontanea o natural (Alberio \& Butler, 2001).

- Estrógenos: En combinación con la progesterona crean una retroalimentación negativa para la $\mathrm{GnRH}$, causando la autodestrucción de los folículos circundantes, pero si son administrados solo con la presencia de un folículo dominante y en ausencia de un cuerpo lúteo, crean el comportamiento de celo e intervienen en la inducción de la ovulación.

- eCG: Gonadotropina coriónica equina), se usa en varios protocolos de sincronización buscando potencializar la acción LH, con el fin de sincronizar la ovulación y aumentar el tamaño del folículo dominante.

\section{Protocolos}

Un estudio realizado por Saldarriaga (2009), plantea la división de los protocolos para la IATF en: los que utilizan combinaciones de $\mathrm{GnRH}$ y prostaglandina F2 $\alpha$ PGF, llamados protocolos Ovsynch y los que utilizan dispositivos con progesterona $\mathrm{P} 4$ y estradiol conocidos como de control del desarrollo folicular.

\section{Protocolo Ovsynch}

Este protocolo es ampliamente usado para sincronización de vacas ciclando ya que provoca ovulación, generalmente sin presentación del celo, por cual es necesario cumplir y respetar los tiempos para hacer IATF. En el protocolo Ovsynch (Figura 1) la GnRH provoca que el folículo más grande ovule o regrese, iniciando una nueva onda folicular; 7 días después al aplicar la PGF2 $\alpha$ se provoca luteolisis y al día 9 la segunda inyección de GnRH induce la ovulación del nuevo folículo; dentro de las próximas 24 h se debe inseminar (Morales \& Cavestany, 2012).

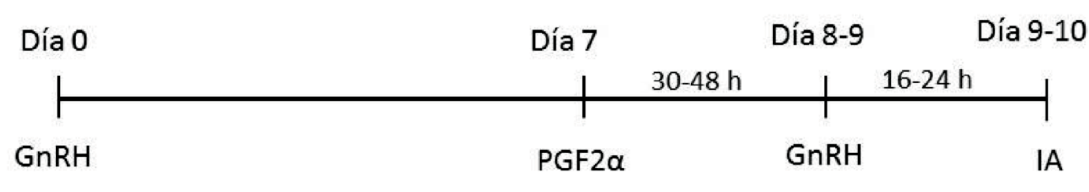

Figura 1. Protocolo Ovsynch:

Fuente: Morales \& Cavestany (2012). 
Estudios recopilados de Morales \& Cavestany (2012), han determinado que los resultados con la aplicación de este protocolo han sido variados de entre $9 \%$ al $37 \%$, por lo cual se han proporcionado a partir de la práctica otras alternativas para complementarlo, como por ejemplo el Cosynch (Figura 2), el cual tiene el agregado de progestágenos P4, la administración de P4 durante 7 días junto con la inyección de $\mathrm{GnRH}$ mejora los porcentajes de preñez entre un $50 \%$ y un $55 \%$ en comparación con vacas en anestro tratadas con el Ovsynch tradicional. Dentro de las variaciones se encuentra con la ventaja de una sesión menos de manejo en los animales, ya que la IATF se establece a la misma vez que la segunda aplicación de la GnRH (Dejarnette \& Nebel, s.f).

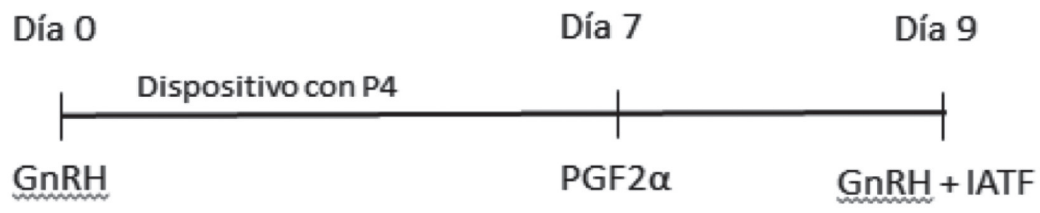

Figura 2. Protocolo Cosynch

Fuente: Morales \& Cavestany (2012); Dejarnette \& Nebel (s.f).

Otra de las variantes del protocolo Ovsynch se basa en la pre-sincronización o Presynch (Figura 3), el cual consiste en 2 aplicaciones de PGF2 $\alpha$ con 14 días de intervalo, la segunda inyección debe ser aplicada 14 días antes del OvSynch o CoSynch, si se desea que las inyecciones sean dadas el mismo día de la semana, o la segunda inyección de PGF2 $\alpha$ a 11 o 12 días antes del OvSynch o CoSynch, si se desea maximizar la tasa de concepción (Dejarnette \& Nebel, s.f). El objetivo con este protocolo radica en tener las vacas en un estado similar del celo al iniciar el protocolo de sincronización para IA, aunque es importante resaltar que su uso es efectivo para aumentar la fertilidad en vacas cíclicas (Morales \& Cavestany, 2012).

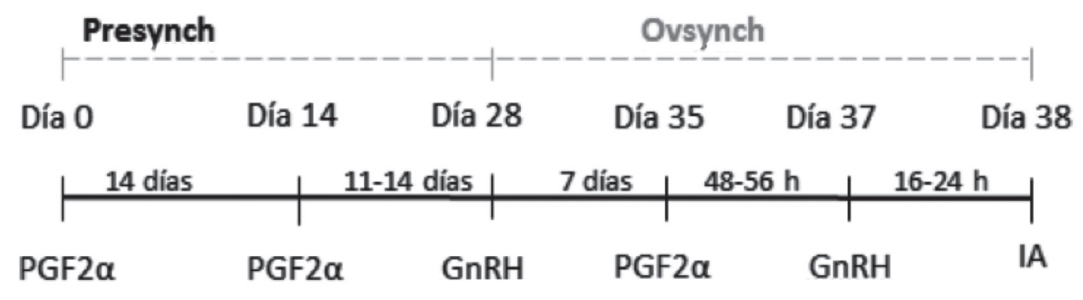

Figura 3. Protocolo Presynch:

Fuente: Morales \& Cavestany (2012).

\section{Protocolos para control del desarrollo folicular}

Recopilación de información de estudios y práticas realizadas en el ganado bovino con anestro, ha direccionado a la creación de tratamientos que mejore esta situación, actualmente en el mercado se ofrecen dispositivos eficientes que liberan Progesterona $\mathrm{P} 4$ y que son mantenidos en la vagina por un período de 7 a 8 días (Saldarriaga, 2009). Morales \& Cavestany (2012), resaltan la función de este protocolo, el cual consiste en imitar una fase luteal corta, producida previo al reinicio de la actividad sexual cíclica posparto, con el fin de mejorar los porcentajes de preñez en animales inseminados artificialmente y evitando la formación de un cuerpo lúteo de vida corta, lo que contribuye a que el cuerpo lúteo de la siguiente ovulación tenga una actividad normal, permitiendo el desarrollo y el mantenimiento de la preñez, también sugierenen base a estudios previos, que la tasa de éxito de los protocolos con progestágenos o P4 sola es variable entre el 50\% al $70 \%$, ya que depende del intervalo parto a tratamiento y la causa del anestro. 
Igualmente estudios de Bó et al., (2008), indica que los productores de leche de todo el mundo que han utilizaso estos protocolos han obtenido tasas de preñez entre 35 y 55\%, las cuales estuvieron influenciadas por la condicion corporal, los dias en lactancia de los animales sincronizados y la produccion de leche de las vacas. Asi mismo se puede inferir sobre los resultados que se desean obtener a la hora de la aplicación de la IATF, los cuales se relacionan con la selección de los animales que se van a tratar, hecho que resalta la importancia del manejo de registros y la palpación rectal por personal calificado.
El protocolo con dispositivo impregnado de progesterona junto con estradiol (Figura 4), dentro de su tratamiento más utilizado consiste en administrar $2 \mathrm{mg}$ de benzoato de estradiol (BE) por vía intramuscular (IM) junto con la implantación del dispositivo en el día 0 del tratamiento; en el día 7 u 8, se extrae el implante y se aplica prostaglandina PGF2 $\alpha-$ IM y 24 h después se administra $1 \mathrm{mg}$ de BE- IM; la IATF se realiza entre las 52 y $56 \mathrm{~h}$ del retiro del dispositivo (Saldarriaga, 2009).

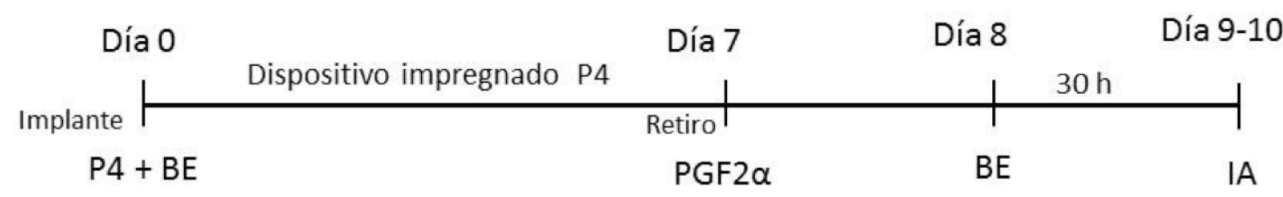

Figura 4. Protocolo Dispositivo impregnado con progesterona junto con estradiol.

Fuente: Saldarriaga (2009).

También se manejan tratamientos con dispositivos de liberación de progesterona, estradiol y Gonadotropina Coriónica equina eCG, indicados para las vacas con buena producción de leche y que presentan anestro posparto, el cual causa un aumento en el intervalo entre partos y afecta de manera negativa el desempeño reproductivo y productivo, experimentos de Bó et al., (2008), reportan tasas de preñez de $52 \%$ en grupos tratados con P4+BE+eCG, afirmando que no se detectan diferencias significativas con los tratamientos sin eCG que maneja un porcentaje del $51 \%$.

En cambio estudios de Morales \& Cavestany (2012), reportan resultados con un porcentaje de concepción del $70 \%$ y $64 \%$ de preñez en vacas para carne, al combinar P4 + eCG + PGF2 $\alpha$, logrando aumentar la sincronía y los porcentajes de concepción en la inseminación subsiguiente, haciendo más satisfactorio y rentable la IATF. También se indica que la adición de eCG en los protocolos de $\mathrm{P} 4$ y BE en animales en anestro se establece como una herramienta útil para mejorar la fertilidad en razas indicus con un alto porcentaje de vacas acíclicas antes de los 60 días posparto.

Espinosa (2010), afirma que para lograr mejores porcentajes de preñez el protocolo de elección P4+BE (Figura 5) es aplicando una dosis de eCG (400 UI) 24 horas después de retirado el dispositivo, logrando una preñez del $62 \%$.

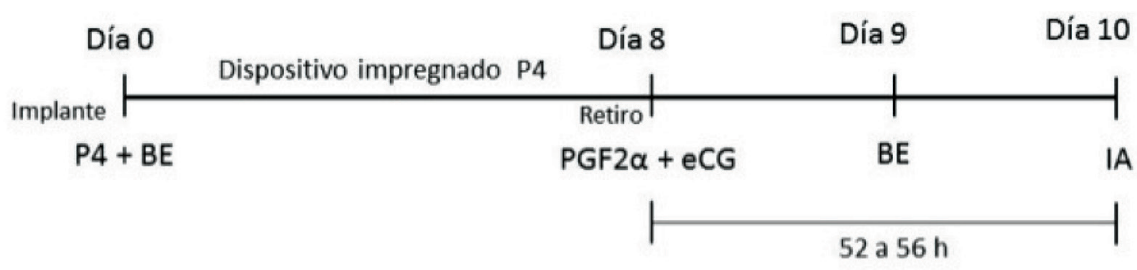

Figura 5. Protocolo P4+BE+eCG.

Fuente: Espinosa (2010). 


\section{Protocolo de resincronización}

El protocolo de resincronización (Figura 6) se toma cuando la vaca muestra celo a los 21 días después de la primera IATF o con el establecimiento de inseminaciones sistémicas en los animales sin la necesidad de detectar celos, acortando los retornos para alcanzar una fertilidades del 55\% al 75\% (Morales \& Cavestany, 2012).

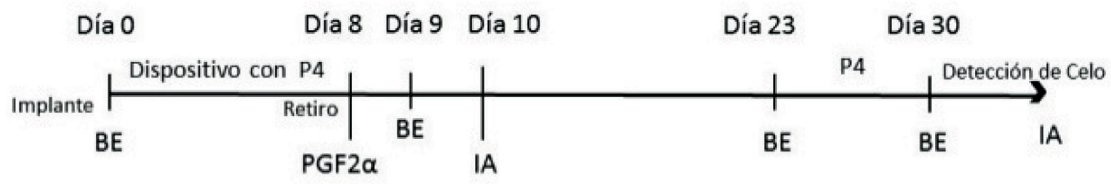

Figura 6. Protocolo de resincronización

Fuente: Bó et al. (2008).

Bó et al. (2008) expresan opinión sobre la ventaja de la resincronización en donde se espera que las vacas regresen al celo en un periodo de tiempo definido, determinando asi que se debe realizar observaciones en campo con más precisión. Asi mismo, la desventaja del programa indica que requiere de tratamientos hormonales continuos y el éxito depende de la eficacia de la detección del celo, lo que incrementa los costos de producción.

Análisis de costo beneficio: programas de IA-IATF Estimaciones propuestas por González (2005), con respecto a los días abiertos (DA), días vacíos (DV) e intervalo entre partos (IEP) para las ganaderías doble propósito (DP) y mestizas en los días de lactación (DEL), la meta ideal, práctica y rentable es obtener una cría al año, en vacas mestizas, la tasa de vacas preñadas con más de 150 DEL varía entre 26,4 y $37,8 \%$, actualmente en las ganaderías se presentan dificultades para lograr estas metas. El manejo de registros evidencia estas dificultades y direcciona las alternativas de solución.

El aumento en DEL $>150$ d demuestra una baja eficiencia reproductiva para las empresas ganaderas que repercutirán en pérdidas económicas productivas. González (2005), ha relacionado en su estudio: "que recomienda el servicio de los animales a partir de 60-75d DEL, ya que antes de los $60 \mathrm{~d}$ se considera un riesgo para la fertilidad, si $60 \%$ de la preñez ocurre entre 60-150d, eso significa que el $40 \%$ tiene DV>150 DEL y lactaciones más largas. Un animal inseminado después de 120 d posparto mantendrá IEP, DEL y lactaciones prolongadas lo que disminuye la rentabilidad". A nivel administrativo de la empresa ganadera se busca reducir los DV, se considera que vacas vacías $>120$ d en razas lecheras las pérdidas económicas están alrededor de 15 mil pesos, mientras que en ganado de carne están alrededor de los 5 o 7 mil pesos por día, si se hace la conversión en animales de mayor producción y con más días vacíos, la rentabilidad de la ganadería disminuye y producen pérdidas para el productor.

El manejo de la IA y la implementación de programas de IATF es una alternativa económicamente viable, la cual produce retornos económicos, basados fundamentalmente en la diferencias de $\mathrm{kg}$ obtenidos al destete (Cutaia et al., s.f), y control en los intervalos entre partos. Esta diferencia ocurre básicamente por la incorporación del mejoramiento genético a nivel productivo y reproductivo, brindando rentabilidad en la empresa ganadera, con mejores producciones en cuanto a su calidad y cantidad (leche) y menos tiempo (carne). En las Tablas 2 y 3 se muestra estudio realizado por Cutaia et al., (s.f), donde se mide la ganancia de peso de terneros con IATF y se compara con los manejados a partir de monta natural, en donde se evidencia el valor genético con relación a la ganancia de peso. 
Tabla 2. Diferencia de peso al destete de terneros machos nacidos por IATF o servicio natural

\begin{tabular}{l|c|c}
\hline & Peso al destete $\mathbf{( k g )}$ & Peso ajustado 205d $\mathbf{( k g )}$ \\
\hline IATF & 211,4 & 201,1 \\
\hline Servicio monta natural & 175,4 & 184,6 \\
\hline Diferencia & 36 & 16,5 \\
\hline
\end{tabular}

Fuente: Cutaia et al. (s.f)

Tabla 3. Diferencia de peso al destete de terneras hembras nacidas por IATF o servicio natural.

\begin{tabular}{l|c|c}
\hline & Peso al destete $\mathbf{( k g )}$ & Peso ajustado 205d (kg) \\
\hline IATF & 196,8 & 185,0 \\
\hline Servicio monta natural & 163,6 & 174,1 \\
\hline Diferencia & 33,2 & 10,9 \\
\hline
\end{tabular}

Fuente: Cutaia et al. (s.f)

Cutaia et al., (s.f), a través de este estudio demuestran que "es posible mejorar los índices productivos en una ganadera de cría aplicando un programa de IATF al comienzo del servicio". Con estos datos se resalta la ventaja del mejoramiento genético desde el inicio del desarrollo productivo del ganado bovino en cuanto a ganancia de peso y producción de carne, lo cual repercute en tiempo y calidad según las características del semen de elección para el manejo en la empresa ganadera.

\section{Relación de costos IA-IATF}

- Vitaminas y minerales como coadyuvantes reproductivos: en algunas vacas para la IA tradicional se recomienda aplicar vitaminas con el fin de que los ovarios tengan una mejor actividad cíclica y así lograr que las vacas entren en celo más rápido. Basado en las deficiencias minerales y de vitaminas dentro de la alimentación.

- Pajillas: en el mercado se pueden encontrar variaciones según la calidad, que van desde los $\$ 15.000$ pesos, hasta precios superiores a $\$ 300.000$ pesos en pajillas de semen importado y semen sexado.

- Sincronización: el costo varía según el protocolo de elección por el profesional, de acuerdo a las condiciones de cada animal y manejo de la empresa ganadera. El protocolo de elección para este análisis se basa en $\mathrm{P} 4+\mathrm{BE}+\mathrm{eCG}$.

- Mano de obra: La mano de obra del inseminador por cada inseminada cuesta alrededor de $\$ 20.000$ pesos. Todo el análisis se muestra en la Tabla 4.

Tabla 4. Relación de costos entre IA tradicional y la IATF.

\begin{tabular}{l|c|c}
\multicolumn{1}{c}{ IA vs IATF } & IA tradicional (\$) & IATF (\$) \\
\hline CalfosvitSe ${ }^{\circledR}$ & $24.200=$ & \\
\hline Valor pajilla/animal & Desde 25.000 hasta $300.000=$ & $60.000=$ \\
\hline Valor sincronización/animal & & $31.000=$ \\
\hline Total sincronización por animal & & $91.000=$ \\
\hline Valor resincronización & $20.000=$ & $4.000=$ \\
\hline Mano de obra inseminador & & $20.000=$ \\
\hline En 10 vacas & & $910.000=$ \\
\hline
\end{tabular}


Los costos relacionados previamente no son exactos, ya que en el programa de IA-IATF se encuentran variaciones debido a las condiciones y estado de los animales, manejo del semen en su aplicación, condiciones ambientales y sanitarias, selección de protocolos, entre otras.

La elección del protocolo depende de las condiciones de los animales, presupuesto de inversión y criterio del profesional que aplique y maneje la IA-IATF.

El riesgo en aplicar y manejar la IA tradicional en vacas cíclicas, depende de la detección del celo y calcular el tiempo exacto para inseminar, por lo cual se ha expresado en muchas experiencias que la probabilidad de preñez es baja. Fallar en la primera IA acarrea aumentar los días abiertos o días vacíos incrementando así el intervalo entre parto y repercutiendo en pérdidas para el productor. Analizando el contexto de la empresa ganadera es más rentable el manejo de la IATF para el mejoramiento genético y mantener buenos parámetros productivos y reproductivos. Como ya se mencionó anteriormente un día vacío en un sistema de producción tiene un costo aproximadamente de entre 15 mil y 7 mil pesos por día para producción de leche y carne respectivamente; El promedio de días abiertos en la ganadería colombiana esta >170d según informe del PEGA 2019 (FEDEGAN-FNG, 2006), reducir este parámetro con la aplicación de la IATF beneficiaria satisfactoriamente la rentabilidad de sector ganadero.

\section{Conclusiones y recomendaciones}

Van Arendonk (2011), expresa que: La primera técnica reproductiva que tuvo un gran impacto en los sistemas de cría de animales fue la inseminación artificial Al, junto con el registro de pedigrí y el registro de leche, para obtener estimaciones exactas en resaltar los valores productivos de los toros en los sistemas de producción de carne y/o leche; los toros contribuyen a la difusión del mejoramiento genético, heredando las características productivas a sus hijas y aportando así una alternativa económica atractiva para la generación de progreso en el sector ganadero.
Dentro de las desventajas que se cuenta en la aplicación de biotecnologías como la IA y la IATF, desde un punto de vista genético, la respuesta depende del grado de interacción genotipo por medio ambiente generando variabilidad de la respuesta o resultados esperados los cuales se verán reflejados a largo plazo, y la endogamia (Nicholas, 1996), lo cual sigue siendo objeto de estudio y estimación dentro del contexto de mejoramiento genético, se espera que con la importación y uso de nuevas generaciones de semen extranjero y manejo de registros se llegue a una disminución de este riesgo, lo cual hasta el momento no ha presentado mayores avances. La inversión de la IA, garantiza en el tiempo mejorar genéticamente la ganadería, por medio de los cruzamientos entre animales comerciales a partir de la aplicación y el manejo de las biotecnologías, determinando los porcentajes raciales de Bos taurus y Bos indicus, para la selección del semen probado y comprobado hacia una genética productiva racialmente, directamente para cada sistema de producción ya sea de carne, leche y/o cría.

\section{Literatura citada}

1. Alberio, R. H. \& Butler, H. (2001). Sincronización de los celos en hembras receptoras. En: Palma, G. A. (Editor). Biotecnología de la reproducción. Instituto Nacional de Tecnología Agropecuaria, Argentina, pp. 61-76.

2. Araujo, R. (2011). Stress calórico en vacas lecheras. Engormix. Lecheria. Recuperado de http://bit. ly/2tUStdK

3. Bavera, G. A. \& Peñafort, C. (2005). Condición corporal (CC). Sitio argentino de producción animal, FAV UNRC. Recuperado de: http://bit.ly/2v9Vd6Q

4. Bó, G. A., Cutaia, L. E., Souza, A. H. \& Baruselli, P. S. (2008). Actualización sobre protocolos de IATF en bovinos de leche. Proceedings of the 3th International Symposium on Animal Reproduction Applied. Londrina, Brazil, 95- 110.

5. Campero, C. M. (2000). Las enfermedades reproductivas en los bovinos: ayer y hoy. Revista de la Academia Nacional de Agronomía y Veterinaria, 53, 88-112.

6. Caraviello, D. Z. (2004). Tópicos de Fertilidad en Vacas de Alta Producción. Instituto Babcock Universidad de Wisconsin. Reproducción y Genética N .611.

7. Carvajal, A.M. \& Kerr, B. (2015). Factores genéticos que influencian la composición de la leche bovina. Instituto de Investigaciones Agropecuarias, INIA Remehue. Recuperado de http://bit.ly/2uLh8Qz 
8. Cutaia, L., Veneranda, G. \& Bó, G. (s.f). Análisis de costo beneficio: programas de inseminación artificial a tiempo fijo y servicio natural. Instituto de Reproducción Animal Córdoba (IRAC), Universidad Católica de Córdoba.

9. Dejarnette, M. \& Nebel, R. (s.f.) Protocolos de sincronización para vacas lecheras. Select Reproductive Solutions. Recuperado de http://www.selectsires.com/ dairy/spanresources/0708_spanish_dairycow.pdf

10. Donato, M. (s.f.). Los registros en la inseminación artificial. Universidad de Córdoba. Recuperado de http:// www.infocarne.com/bovino/inseminacion2.asp

11. Espinosa, M. (2010). Efecto de diferentes protocolos para IATF sobre las tasas de preñez aplicados en ganado lechero. Especialidad en reproducción bovina. Instituto de reproducción animal Córdoba. (IRAC), Córdoba, Argentina.

12. FEDEGAN-FNG. (2006). Plan Estratégico de la Ganadería Colombiana 2019. 1ra Ed. Sanmartín Obregón \& Cía, Bogotá, Colombia.

13. Foote, R. (2002). The history of artificial insemination: Selected notes and notables. American Society of Animal Science

14. Fuentes S, D. (s.f.). Inseminación artificial: ¿De qué depende su éxito o fracaso? Actualidad Ganadera. Recuperado de $h t t p: / / b i t . l y / 2 v c D C L c$

15. Galeano, A. P. (2010). Evaluación genética del recurso animal de los sistemas de producción de bovinos en doble propósito en Colombia. (Tesis de Magíster). Universidad nacional de Colombia. Bogotá, Colombia.

16. Gallego, M. I. (1998). Reproducción del ganado lechero. En: Ruiz, M. E., Rivera, B. \& Ruiz, A. (Editores). Reproducción animal: métodos de estudio en sistemas. IICA/RISPAL. Costa Rica.

17. Gambini, B. B. (2009). Las buenas practicas ganaderas (BPG) mejoran la producción, pero ¿Mejoran el bolsillo? Agro Enfoque, 23, (165), 54-57.

18. Gasque, R. (2008). Enciclopedia bovina. 1ra Ed. FMVZ. Capítulo 8, Mejoramiento Genético en bovinos. México, pp. 267-285.

19. González, C. (2005). Calcule los días en producción y la tasa de preñez. En: Manual de Ganadería Doble Propósito. Ediciones Astro Data, S.A. Maracaibo-Venezuela. VIII (1): 257-264.

20. Martínez, N. (s.f.) Alternativa para mejorar la inseminación artificial. Suganado.com. Recuperado de http:// suganado.com/noticias_detalle.php?/d_Noticia=86
21. Molina, R., Sánchez, H., Uribe, J. \& Atzori, A. (2016). Efecto de la edad al primer parto y los días abiertos en un bovino doble propósito sobre la huella hídrica y de carbono. Revista de Investigación Agraria y Ambiental, 7 (2), 107-119. Recuperado de: http://hemeroteca. unad.edu.co/index.php/riaa/article/view/1561/1908

22. Montenegro, S. \& Hernández, Y. (2015). Biotecnología aplicada al desarrollo agropecuario colombiano. Revista de Investigación Agraria y Ambiental, 6 (2) 97108. Recuperado de: http://hemeroteca.unad.edu.co/ index.php/riaa/article/view/1408/1732

23. Morales, J. T. \& Cavestany, D. (2012). Anestro posparto en vacas lecheras: tratamientos hormonales. Veterinaria, Vol. 48 (185-188), pp. 19-27.

24. Motta, J. L., Waltero, I., Abeledo, M. A. \& Fernández, O. (2012). Estudio retrospectivo de agentes infecciosos que afectan la reproducción bovina en el departamento del Caquetá, Colombia. Revista Salud Animal, 34 (3), 159-164.

25. Nicholas, F.W. (1996). Genetic improvement through technology. Animal Reproduction Science, 42, 205-214.

26. Restrepo, G. (2008). Biotecnologías reproductivas aplicables a la producción bovina en Colombia. Grupo de investigación en biotecnología animal-GIBA. Medellín, Colombia.

27. Ruiz, E. (2013). Mejoramiento genético en ganado de carne para el trópico. Guía técnica-Agrocampo, Perú. Recuperado de http://www.agrobanco.com.pe/datal uploads/ctecnica/018-c-ganado.pdf

28. Saldarriaga, E. F. (2009). Análisis comparativo entre inseminación artificial a tiempo fijo e inseminación artificial a celo detectado, con sus variables económicas y reproductivas. (Informe de práctica profesional). Corporación Universitaria Lasallista, Caldas.

29. Santori, R. (2009). Factores nutricionales que afectan el desempeño en programas reproductivos en bovinos de carne y de leche. Conferencia dictada en el $8^{\circ} \mathrm{Sim}$ posio Internacional de Reproducción Animal del IRAC, Córdoba, Argentina.

30. Uribe, H. (s.f.). Mejoramiento Genético de Ganado de Leche. Instituto de Investigaciones Agropecuarias. Centro Regional de Investigación Remehue. Boletín Inia $\mathrm{N}^{\circ} 148$.

31. Van Arendonk, J.A.M. (2011). The role of reproductive technologies in breeding schemes for livestock populations in developing countries. Livestock Science, 136, 29-37.
Conflicto de Intereses

Los autores declaran no tener ningún conflicto de intereses

Recibido: Marzo 10 de 2017 Aceptado: Abril 17 de 2017 